\title{
EDITORIAL
}

\section{Allied health professionals: fundamental to the transplant multidisciplinary team}

For referencing Montgomery E. Allied health professionals: fundamental to the transplant multidisciplinary team. Transplant Journal of Australasia 2021;30(2):3.

DOI https://doi.org/10.33235/tja.30.2.3

Modern transplant-related healthcare is a team venture and allied health professionals are fully integrated members of the transplant team. Allied health professionals are involved throughout the transplant journey from initial evaluation to the post-transplant period, including inpatient, outpatient and long-term care of recipients. Allied health is a rapidly growing field and area of need. As the criteria for transplant candidates continue to expand, leading to an increase in more complex recipients, the crucial role of allied health in managing transplant patients has never been more apparent.

Allied health providers range from dietitians and psychologists to podiatrists, to name just a few. All of our allied health colleagues play a crucial role in addressing various aspects of people's health and delivering services to improve the health and wellbeing of our transplant candidates and recipients. At times the work of allied health professionals may go unrecognised or underappreciated. In this edition we aim to highlight and pay tribute to the hard work of our allied health team members.

Our guest editorial is written by Kirsty McDowall, senior dietitian with the South Australian Liver Transplant Unit, Flinders Medical Centre. Kirsty walks us through her successful career and explores the multifaceted nature of her role.

The field of lung transplantation has seen rapid growth and progress in the pharmaceutical management of patients in recent years. This has led to more complex medication regimes, a greater potential for adverse effects and interactions, and an ever-increasing cost. Lung transplant pharmacists play a central role in continuing to deliver evidence-based healthcare. Lung transplant pharmacists Sarah Hitchen, Rachel Thorson and Jeanie Misko from Fiona Stanley Hospital in Western Australia describe how they overcame the challenges of ongoing transplant-related medications while introducing a chemotherapy regime in a lung transplant patient. Their article emphasises the critical contribution pharmacists make to the multidisciplinary team.

We all understand how important it can be to both donor families and transplant recipients to correspond. Carla Van Weerdenburg from the Organ and Tissue Authority discusses the recent changes to the National Standard Operating Procedure (SOP) for managing the exchange of correspondence between donor families and transplant recipients. The updated SOP improves the process of managing correspondence between donation agencies and transplant units and better reflects the needs and expectations of both donor families and transplant recipients.

Mallory Hall and Georgia Wakefield, dietitians from St Vincent's Hospital in Sydney, describe the complex role of the heart and lung transplant dietitian. From pre-operative management to ongoing post-operative care, the dietitian is key to patients returning to an optimal quality of life. In their article, Mallory and Georgia present their exciting upcoming research project, The impact of pre-operative nutrition support in heart and lung transplant patients: examining feasibility and acceptability.

Vicki Jermyn, clinical nurse consultant in liver transplantation at The Children's Hospital Westmead, has dedicated a majority of her career to the care of paediatric liver transplant recipients. As Vicki moves into retirement, Janine Sawyer from The Children's Hospital Westmead recounts the incredible career highs, including some more comical moments, in tribute to Vicki's hard work and dedication over the years. We wish Vicki all the best in her retirement and have no doubt she will be greatly missed!

As much of the country continues to grapple with the ongoing impact of COVID-19 restrictions, I hope that everyone is taking time to care for themselves. Remember to touch base with family, friends and colleagues. It is important to draw strength from each other during these times and get through this together.

Something positive to look forward to is the TNA National Conference to be held in Adelaide from 20-22 October this year. Full details are available on the TNA website www. transplantnurses.org.au. Registration is open and available to attend face to face or online. I encourage everyone to make use of this exciting professional development opportunity. After all, we could all use a bit of excitement and something different in our lives at the moment!

Finally, as we head to our final edition for the year this December, I encourage and welcome anyone considering contributing to the journal. Why not use some of those hours in lockdown to write up an interesting case study or research project? The TJA Editorial Board and I provide ongoing support for first-time authors, and all other authors if required. Get in touch today if you have an idea you would like to see in print this December - tjaeditor@ transplantnurses.org.au 